\title{
Endogenous Klebsiella Endophthalmitis Associated with Liver Abscess: First Case Report from Iran
}

\author{
A.R. Dehghani A. Masjedi F. Fazel H. Ghanbari \\ M. Akhlaghi N. Karbasi \\ Ophthalmology Ward, Feiz Hospital, University of Medical Science, Isfahan, Iran
}

\section{Key Words}

Klebsiella pneumoniae $\cdot$ Endophthalmitis · Liver abscess

\begin{abstract}
Purpose: To report the first case of endogenous Klebsiella endophthalmitis associated with liver abscess in Iran.

Case Report: A 79-year-old man was referred to our hospital due to severe pain and visual loss in the left eye. On physical examination, conjunctival hyperemia, corneal edema, hypopyon and severe vitreous cellular reaction were identified in the left eye; however, yellowish conjunctival discoloration was more apparent in the right eye. Abdominal CT scan showed a right liver lobe abscess that was confirmed by sonographically guided percutaneous liver mass biopsy. Blood, vitreous and liver mass aspirate cultures revealed Klebsiella pneumoniae growth. The patient was thus diagnosed with endogenous Klebsiella endophthalmitis secondary to bacteremia associated with liver abscess.
\end{abstract}

Conclusion: This report suggests that, rather than being confined to Taiwan, endogenous endophthalmitis secondary to a liver abscess due to K. pneumoniae may be a global problem. Therefore, physicians should be aware of the possibility of endophthalmitis whenever a patient with $K$. pneumoniae liver abscess complains of ocular symptoms.

\section{Introduction}

Endogenous bacterial endophthalmitis results from blood-borne spread of bacteria. Remote, non-ocular diseases such as endocarditis, gastrointestinal disorder, urinary disease and meningitis may be the source of bacteria. Immunocompromised states such as diabetes mellitus, malignancy and chemotherapy are associated with a reduced host 
defense and are risk factors for the development of endogenous endophthalmitis [1]. Endogenous endophthalmitis in immunocompetent patients is rare [2].

A comprehensive search of the literature did not reveal any reported cases of endogenous endophthalmitis secondary to Klebsiella pneumoniae in patients with liver abscesses in Iran. In this report, we describe a case of endogenous Klebsiella endophthalmitis as one of the initial presentations of a liver abscess.

\section{Case Report}

A 79-year-old man was referred to our hospital due to a 2-day history of severe visual loss in the left eye associated with pain. In addition, the patient presented with fever and anorexia and had a positive history of hypertension, without any other systemic disorder such as diabetes mellitus. Two years before admission, the patient underwent a phacoemulsification cataract surgery on both eyes.

General physical examination revealed fever and tachycardia. Other organ examinations were normal except for mild right upper quadrant abdominal pain. On ophthalmologic examination, the visual acuity was 10/10 in the right eye and hand motion in the left eye, in addition to a $3+$ relative afferent pupillary defect. Red reflex was observed in the right eye, but not seen in the left eye. In addition, the pupil was reactive in the right eye but completely nonreactive in the left eye. The extraocular muscle function was normal, with no obvious deviation in ocular alignment. On slit lamp examination, the right eye appeared normal except for a mild yellowish discoloration of the conjunctiva. Examination of the left eye revealed that the lid and lashes were normal. The conjunctiva was severely hyperemic, with dilation of conjunctival vessels and ciliary injection, and the cornea was edematous, with keratic precipitates in the corneal endothelium. The anterior chamber was filled with fibrin and showed a 4-mm hypopyon. The iris was irregular due to posterior synechia formation. Additionally, the lens was fibrin coated and the vitreous had a $4+$ cellular reaction. The condition of the retina, optic nerve and choroid could not be evaluated because of hazy ocular media. The intraocular pressure was normal in both eyes. A sonography of the left eye demonstrated increased vitreous echogenicity indicating vitreous pus formation, but there was no sign of retinal detachment or posterior segment mass.

The patient was then hospitalized because of suspicion of endogenous endophthalmitis and he underwent intravitreal antibiotic injection (vancomycin $1 \mathrm{mg} / 0.1 \mathrm{cc}$ and ceftazidime $2.25 \mathrm{mg} / 0.1 \mathrm{cc}$ ), followed by deep vitrectomy $6 \mathrm{~h}$ after intravitreal injection due to rapid progression of endophthalmitis and to a decline in visual acuity during this period. He also received systemic antibiotics (vancomycin and gentamicin) for 2 weeks.

An additional evaluation revealed that the patient had leukocytosis (neutrophilia). The erythrocyte sedimentation rate was $80 \mathrm{~s}$ and the C-reactive protein level was $3+$. A liver function test revealed hyperbilirubinemia (total bilirubin $=6 \mathrm{mg} / \mathrm{dl}$, direct bilirubin $=2 \mathrm{mg} / \mathrm{dl}$ ) with normal liver enzyme levels $(\mathrm{AST}=41 \mathrm{IU} / \mathrm{l}, \mathrm{ALT}=44 \mathrm{IU} / \mathrm{l})$. There was no evidence of viral hepatitis. Blood and vitreous sample cultures showed K. pneumoniae growth. Urine analysis and urine culture were both normal. Abdominal CT scan showed an irregular hypodense lesion in the right liver lobe, with central necrosis and irregular wall enhancement (fig. 1). Other structures, such as the pancreas, kidney, and bladder, were all normal. This finding was suggestive of a liver abscess, which was confirmed by a sonographically guided percutaneous liver mass biopsy and drainage. K. pneumoniae was sensitive to ciprofloxacin and kanamycin. Thus, we changed the first antibiotic therapy to ciprofloxacin.

Now, one month after the treatment, the patient's visual acuity is poor. He presents with light perception vision with pigment deposition of the corneal endothelium and no signs of retinal detachment. Diffuse nerve fiber layer loss and 2+ optic pallor are detected in fundoscopy. 


\section{Discussion}

Septic metastatic endophthalmitis is a rare but serious disease which occurs when bacteria cross the blood-ocular barrier and multiply within the eye. The majority of patients diagnosed with endogenous bacterial endophthalmitis are initially misdiagnosed and may have an underlying disease known to predispose to infection [3]. The most common Gram-positive organisms are Staphylococcus aureus, group B streptococci, Streptococcus pneumoniae and Listeria monocytogenes. The most common Gram-negative organisms are Klebsiella spp., Escherichia coli, Pseudomonas aeruginosa and Neisseria meningitides. Gram-negative organisms are responsible for the majority of the cases reported from East Asian hospitals [4].

K. pneumoniae is an enteric Gram-negative bacillus $[4,5]$. It can cause hospitalacquired infection in debilitated or immunocompromised patients [6]. A new type of this bacterium has emerged in Taiwan, which is typically associated with a communityacquired liver abscess with bacteremia and sepsis [7]. Endophthalmitis complicated the course in $10-12 \%$ of cases [8]. In addition to being particularly common in Taiwan, where more than 900 cases have been reported, this invasive K. pneumoniae disease has also been observed in North America, Europe and Japan [9].

K. pneumoniae liver abscess is known to be related to metastatic endophthalmitis, with most cases clustered in Taiwan [10]. Disseminated intravascular coagulation is also a risk factor for endogenous endophthalmitis in patients with Klebsiella sepsis [11].

In this article, we report the first Iranian case of endogenous endophthalmitis caused by $K$. pneumoniae in a patient with a liver abscess. Diabetes mellitus is the most common comorbid risk factor in this disease [12]. However, our patient had no history of diabetes mellitus. Liver abscess has been reported to be the major source of endophthalmitis caused by Klebsiella [13], but other sources such as renal or prostate abscesses can also contribute to the septic metastasis to the eye $[14,15]$. Our patient did not have other sites for K. pneumoniae as a source for metastasis to the eye.

In this patient, the endogenous endophthalmitis was detected first; searching for the site of the source of the infection then revealed the abscess in the right lobe of the liver. In addition, the blood culture was positive for this organism. Thus, this patient was diagnosed as having endogenous endophthalmitis due to bacteremia, with the liver abscess as the source.

The visual prognosis has been poorly reported in several studies. Diabetes mellitus might be a significant risk factor for the poor visual outcome $[16,17]$. The prognosis of maintaining visual acuity in patients with septic endophthalmitis is poor in spite of an early diagnosis and the timely start of conventional therapeutic procedures because the intravitreous drug concentration remains low after the systemic administration of antibiotics due to the blood-ocular barrier; however, there exists a case report of a successful treatment of endogenous Klebsiella endophthalmitis [18]. The outcome of endogenous bacterial endophthalmitis has not improved in 55 years [3]. In one study, a retrospective review of 289 patients with a clinical diagnosis of pyogenic liver abscess revealed 10 patients (3.5\%) with the complication of endogenous endophthalmitis with the same offending organism, $K$. pneumoniae; among them, 5 patients had no light perception, 1 patient had light perception and 4 patients were able to visualize hand motion only [19]. 


\begin{tabular}{c|l|l|l} 
Case Reports In & $\begin{array}{l}\text { Case Rep Ophthalmol 2011;2:10-14 } \\
\text { DOl: 10.1159/000323449 }\end{array}$ & $\begin{array}{l}\text { Published online: } \\
\text { January 7, 2011 }\end{array}$ & $\begin{array}{l}\text { 2011 S. Karger AG, Basel } \\
\text { ISSN 1663-2699 } \\
\text { www.karger.com/cop }\end{array}$ \\
\hline
\end{tabular}

In the end, physicians should be aware of the possible development of endogenous endophthalmitis in patients with Klebsiella septicemia, especially in diabetics with a hepatobiliary abscess.

\section{Conclusion}

This report suggests that, rather than being confined to Taiwan, endogenous endophthalmitis secondary to a liver abscess due to K. pneumonia may be a global problem.

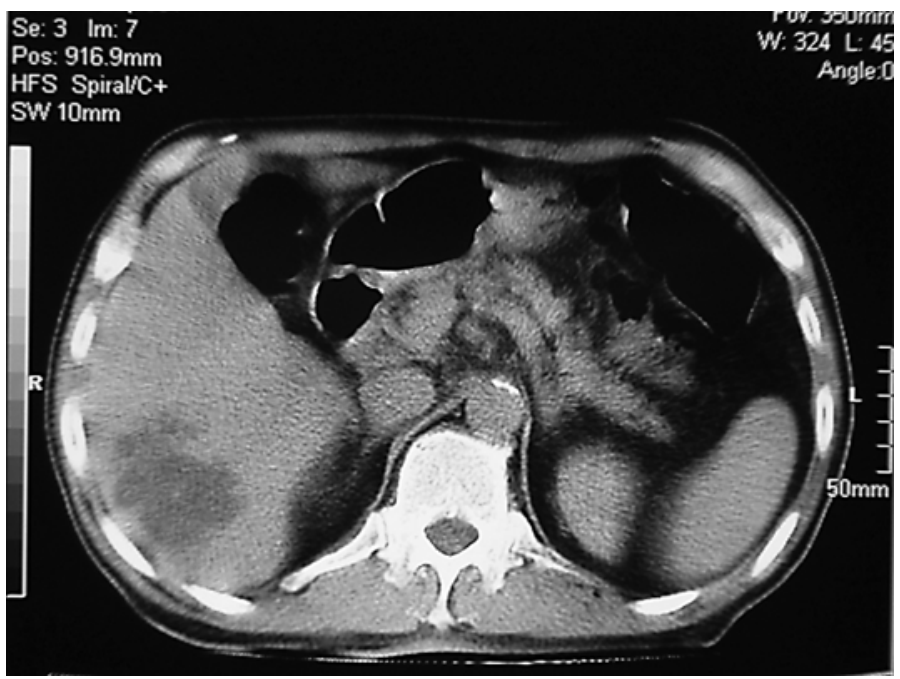

Fig. 1. Right liver lobe abscess.

\section{References}

1 Seok S, Jang YJ, Lee SW, Kim HC, Ha GY: A case of bilateral endogenous Pantoea agglomerans endophthalmitis with interstitial lung disease. Korean J Ophthalmol 2010;24:249-251.

2 Kim SJ, Seo SW, Park JM, Chung IY: Bilateral endophthalmitis as the initial presentation of bacterial meningitis. Korean J Ophthalmol 2009;23:321-324.

- Jackson TL, Eykyn SJ, Graham EM, Stanford MR: Endogenous bacterial endophthalmitis: a 17-year prospective series and review of 267 reported cases. Surv Ophthalmol 2003;48:403-423.

4 Abbott S: Klebsiella, Enterobacter, Citrobacter and Serratia; ; in Murray PR, Baron EJ, Pfaller MA, Tenover FC, Yolken RH (eds): Manual of Clinical Microbiology, 7th ed. Washington, American Society for Microbiology Press, 1999, pp 475-482.

5 Eisenstein BI, Zaleznik DF: Enterobacteriaceae; in Mandell GL, Bennett JE, Dolin R (eds): Principles and Practice of Infectious Diseases, 5th ed. Philadelphia, Churchill-Livingstone, 2000, pp 2294-2309.

6 Podschun R, Ullmann U: Klebsiella spp. as nosocomial pathogens: epidemiology, taxonomy, typing methods and pathogenicity factors. Clin Microbiol Rev 1998;11:589-603. 
7 Wang JH, Liu YC, Lee SS, Yen MY, Chen YS, Wang JH, Wann SR, Lin HH: Primary liver abscess due to Klebsiella pneumoniae in Taiwan. Clin Infect Dis 1998;26:1434-1438.

-8 Liu YC, Cheng DL, Lin CL: Klebsiella pneumoniae liver abscess associated with septic endophthalmitis. Arch Intern Med 1986;146:1913-1916.

-9 Fang CT, Chuang YP, Shun CT, Chang SC, Wang JT: A novel virulence gene in Klebsiella pneumoniae strains causing primary liver abscess and septic metastatic complications. J Exp Med 2004;199:697-705.

10 Connell NT, Thomas IA, Sabharwal AD, Gelbard MA: Klebsiella pneumoniae endophthalmitis with associated hepatic abscess. J Hosp Med 2007;2:442-444.

11 Sng CC, Jap A, Chan YH, Chee SP: Risk factors for endogenous Klebsiella endophthalmitis in patients with Klebsiella bacteraemia: a case-control study. Br J Ophthalmol 2008;92:673-677.

12 Yang CS, Tsai HY, Sung CS, Lin KH, Lee FL, Hsu WM: Endogenous Klebsiella endophthalmitis associated with pyogenic liver abscess. Ophthalmology 2007;114:876-880.

13 Chen YJ, Kuo HK, Wu PC, Kuo ML, Tsai HH, Liu CC, Chen CH: A 10-year comparison of endogenous endophthalmitis outcomes: an east Asian experience with Klebsiella pneumoniae infection. Retina 2004;24:383390.

14 Chuang LH, Song HS, Lee SC, Lai CC, Ku WC: Endogenous Klebsiella pneumoniae endophthalmitis associated with prostate abscess: case report. Chang Gung Med J 2000;23:240-245.

15 Chen KJ, Hwang YS, Wang NK, Chao AN: Endogenous Klebsiella pneumoniae endophthalmitis with renal abscess: report of two cases. Int J Infect Dis 2010;14:e429-e432.

16 Sheu SJ, Chou LC, Hong MC, Hsiao YC, Liu YC: Risk factors for endogenous endophthalmitis secondary to Klebsiella pneumoniae liver abscess. Zhonghua Yi Xue Za Zhi (Taipei) 2002;65:534-539.

-17 Scott IU, Matharoo N, Flynn HW Jr, Miller D: Endophthalmitis caused by Klebsiella species. Am J Ophthalmol 2004;138:662-663.

18 Ishii K, Hiraoka T, Kaji Y, Sakata N, Motoyama Y, Oshika T: Successful treatment of endogenous Klebsiella pneumoniae endophthalmitis: a case report. Int Ophthalmol 2010; E-pub ahead of print.

19 Tan YM, Chee SP, Soo KC, Chow P: Ocular manifestations and complications of pyogenic liver abscess. World J Surg 2004;28:38-42. 